\title{
Cognard Type V intracranial dural arteriovenous fistula presenting in a pediatric patient with rapid, progressive myelopathy
}

\author{
Walter J. Jermakowicz, MD, PhD, Alexander G. Weil, MD, Artyom Vlasenko, MD, \\ Sanjiv Bhatia, MD, and Toba N. Niazi, MD
}

Division of Pediatric Neurosurgery, Nicklaus Children's Hospital, and Department of Neurosurgery, University of Miami Miller School of Medicine, Miami, Florida

\begin{abstract}
Cognard Type V dural arteriovenous fistulas (dAVFs) are a unique type of cranial vascular malformation characterized by congestion of the perimedullary venous system that may lead to devastating spinal cord pathology if left untreated. The authors present the first known case of a pediatric patient diagnosed with a Type V dAVF. A 14-year-old girl presented with a 3-week history of slowly progressive unilateral leg weakness that quickly progressed to bilateral leg paralysis, sphincter dysfunction, and complete sensory loss the day of her presentation. MRI revealed an extensive T2 signal change in the cervical spine and tortuous perimedullary veins along the entire length of the cord. An emergency cranial angiogram showed a Type V dAVF fed by the posterior meningeal artery with drainage into the perimedullary veins of the cervical spine. The fistula was not amenable to embolization because vascular access was difficult; therefore, the patient underwent urgent suboccipital craniotomy and ligation of the arterialized venous drainage from the fistula. The patient's clinical course immediately reversed; she had a complete recovery over the course of a year, and she remains asymptomatic at the 2-year follow-up. This report adds to a growing body of evidence that describes the diverse and unpredictable nature of Type V dAVFs and highlights the need to obtain a cranial angiogram in pediatric patients with unexplained myelopathy and cervical cord T2 signal change on MRI.
\end{abstract}

https://thejns.org/doi/abs/10.3171/2017.3.PEDS16363

KEY WORDS vascular malformation; surgical clipping; quadriparesis; vascular disorders

I NTRACRANIAL dural arteriovenous fistulas (dAVFs) are a heterogeneous group of vascular lesions characterized by abnormal shunting of arterial blood into the venous system of the dura. DAVFs account for only $10 \%-$ $15 \%$ of intracranial vascular malformations. ${ }^{2}$ In pediatric patients, the incidence of a dAVF is lower than in adults, but the clinical course is often more aggressive and with a worse prognosis. ${ }^{6}$ This is especially true when pediatric patients acquire pial intracranial or perimedullary venous drainage through a communication of dural sinuses with normal pial veins. Novel endovascular therapies have improved the treatment options available for children with dAVFs, with good reported outcomes. ${ }^{3}$ Thus, prompt diagnosis of dAVFs in this patient population is necessary to provide adequate therapy and prevent permanent neurological injury. The clinical presentation of dAVFs is highly variable and depends largely on the venous drainage pattern. Patients may be asymptomatic or have clinical mani- festations ranging from headaches or tinnitus to neurological deficits, elevated intracranial pressure, hypertensive encephalopathy, or intracranial hemorrhage. Higher-grade dAVFs, which have cortical venous reflux caused by venous hypertension, have a $10.4 \%$ annual mortality rate in adults. ${ }^{4,8}$

Cognard Type V dAVFs are a subset of cranial dAVFs with spinal venous drainage that cause perimedullary venous congestion (Table 1), leading to spinal cord edema and decreased tissue perfusion. ${ }^{1}$ In adults, these lesions present with progressive ascending myelopathy or bulbar symptoms that may be remote from the site of the fistula. Symptoms usually develop over the course of weeks or months, but several reports have documented rapid progression to complete tetraparesis over the course of days. Type V Cognard dAVFs have a nearly 4:1 predilection for males over females, and the average age at onset of reported cases is 56 years old. To our knowledge, no prior

ABBREVIATIONS dAVF = dural arteriovenous malformation 
TABLE 1. Cognard system for classification of dAVFs

\begin{tabular}{cc}
\hline Cognard Type & \multicolumn{1}{c}{ Clinical Features } \\
\hline I & Confined to sinus, no cortical venous reflux \\
II & $\begin{array}{c}\text { Confined to sinus, reflux into sinus without (Ila) or } \\
\text { with (Illb) cortical venous reflux }\end{array}$ \\
\hline III & Drain directly into cortical veins, not into sinus \\
\hline IV & Drain directly into cortical veins, w/ venous ectasia \\
\hline V & Spinal perimedullary drainage \\
\hline
\end{tabular}

The classification is based on venous draining patterns and correlated with clinical course as defined by Cognard et al.

case has been reported in the pediatric population. Most recent reports of dAVF have described a combination of endovascular and surgical therapy. Overall, $60 \%$ of patients experience clinical improvement after surgical or intravascular treatment. ${ }^{4}$

We describe the case of a 14-year-old girl with no prior medical history or predisposing factors who was diagnosed with a Type V dAVF after presenting with rapidly progressive myelopathy. She was treated promptly with angiography and surgical clipping of the fistula, resulting in a full recovery.

\section{Case Report}

History and Presentation

A 14-year-old girl with no significant medical history or recent contact with sick individuals presented with progressively worsening lower-extremity weakness and difficulty with ambulation. The weakness began 3 weeks prior to presentation in the right leg with associated dragging of the right foot, but progressed to the left leg 1 day prior to presentation. The patient initially presented to an outside hospital, where cervical MRI showed intramedullary edema extending from C-3 to C-7 that was concerning for tumor, inflammatory process, or vascular malformation. After 2 doses of dexamethasone with no improvement, the patient was transferred to our hospital.

Physical examination on admission demonstrated an awake, alert patient with no signs of respiratory distress, and it revealed lower-extremity spastic paraplegia and absent sensation below the T-12 level. Rectal tone was present. The results of laboratory evaluations, including white blood cell count, erythrocyte sedimentation rate, and Creactive protein, were within normal limits.

Additional cervical MRI demonstrated intramedullary expansion and diffuse central T2 signal abnormality extending from C-3 to C-7 (Fig. 1), with stable appearance compared with findings on the earlier MR images. Tortuous serpentine veins were noted in the cervical cord trailing down to the thoracic cord. After contrast administration, ventral vermiform enhancement of the cord from C-4 to C-6 was evident. No restricted diffusion was noted. These findings suggested the presence of a dAVF.

\section{Angiography}

The patient showed significant decline over the course of 8 hours after presenting to our hospital, which prompt- ed emergency angiographic evaluation. Prior to the angiographic procedure, her motor function had deteriorated to paraplegia and there was no rectal tone with urinary retention.

Injection of the left vertebral artery revealed a dural fistula along the suboccipital convexity dura mater filled by the left posterior meningeal artery, suggestive of a Cognard Type V dAVF (Fig. 2). A prominent early draining vein was noted that drained across the midline to a tangle of veins $5 \mathrm{~mm}$ in breadth. This venous complex drained inferiorly to prominent dilated serpentine perimedullary veins, visible down to the thoracic cord.

The patient was transferred to the operating room immediately for surgical treatment because access to the fistula via endovascular approaches was hindered by the small size and serpentine course of the feeders. Also, the draining vein was presumed to be easily accessible via a posterior open surgical approach.

\section{Surgical Treatment}

The intubated patient was placed prone using 3-point head pin fixation. Intraoperative monitoring was used with poor potentials distally. A suboccipital craniotomy was performed with C-1 laminectomy to achieve optimal visualization of the dura. Because the angiogram demonstrated that the fistula was on the left side, a Y-shaped incision into the dura was made with the vertical branch toward the right side (Fig. 3). There was minimal bleeding as the midline was crossed, which was well controlled with Weck clips (Teleflex). Once the dura was opened, the fistula was immediately appreciated within the dural leaflet on the right side. An arterialized vein was visible intradurally under the arachnoid (Fig. 4 left). The surgical microscope was introduced, and the arachnoid was opened using microscissors. The location of this arterialized vein did appear to correspond to the fistula, as judged on angiography, but, to ensure isolation of the fistula and not one of the posterior inferior cerebellar arteries, the latter were identified and isolated bilaterally. Once the arterialized vein was identified, a temporary clip was placed to ensure that there were no changes in potentials; the vein was coagulated and transected (Fig. 4 right), and medium-sized clips were placed on either side. The dura was approximated in a primary fashion with no dural graft needed. The bone was attached with a plate-and-screw system. The incision was then closed, and the patient was extubated. She was given a standard dose of postoperative antibiotics, but no steroids were administered during the postoperative period.

\section{Postoperative Course}

The patient awoke from surgery with no new neurological deficits. She began to regain lower-extremity sensory and motor function and rectal function immediately after surgery and was mobilized quickly. MRI performed 6 days after surgery demonstrated cervical cord edema that showed improvement over preoperative MRI, with no evidence of the previously engorged perimedullary veins (Fig. 5).

After a week-long hospitalization with daily physical and occupational therapy, the patient was transferred to 

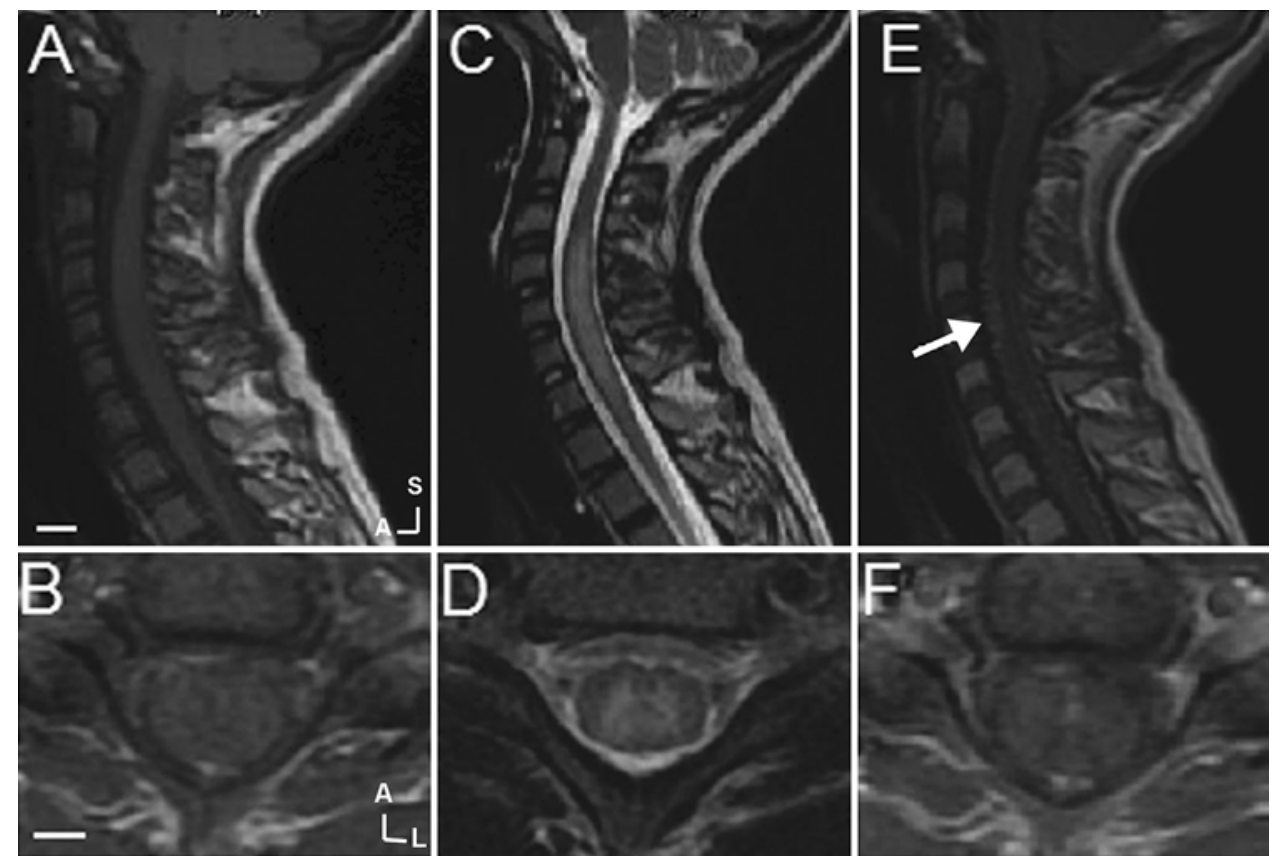

FIG. 1. Preoperative MR images with contrast obtained at presentation. Sagittal (A) and axial (B) T1-weighted images showing medullary expansion extending from C-2 to C-7. Sagittal (C) and axial (D) T2-weighted scans showing abnormal signal enhancement from C-3 to C-7 with associated perimedullary flow voids. Sagittal $(E)$ and axial $(F)$ T1-weighted images postcontrast showing vermiform enhancement in the ventral cervical cord (arrow). Scale bar $=10 \mathrm{~mm}(A, C$, and $E) ; 4 \mathrm{~mm}(B, D$, and $F) . A=$ anterior; $L=$ lateral; $\mathrm{S}=$ superior.
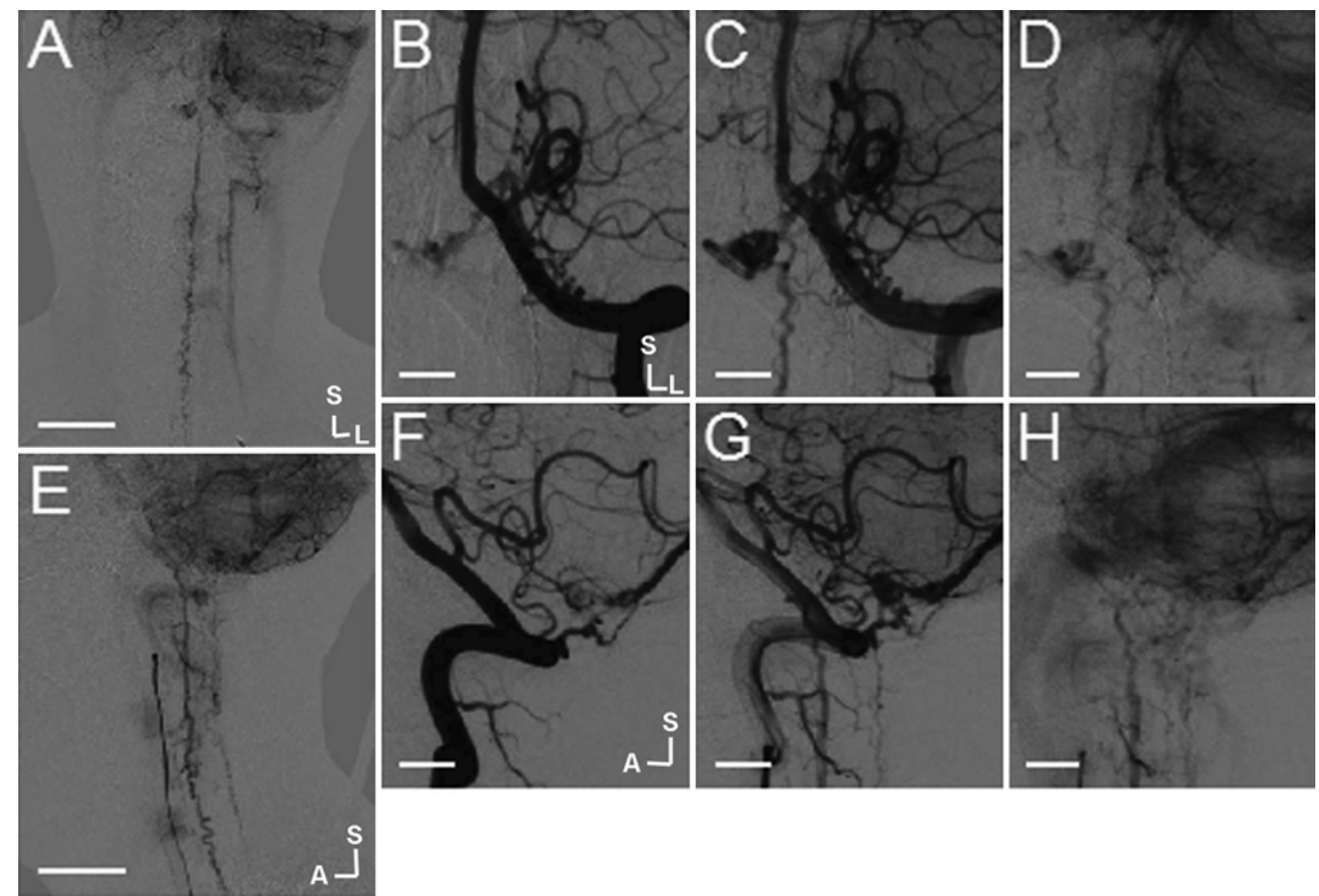

FIG. 2. Angiographic evaluation. A: Anteroposterior view of a left vertebral artery injection showing posterior fossa dAVF with serpentine perimedullary drainage extending the length of the spinal cord. B-D: Serial anteroposterior images magnified to the posterior fossa, showing quick filling of the fistula and draining vein near the midline. E: Lateral view of the left vertebral artery angiogram presented in panel A. F-H: Serial lateral images magnified to show the posterior fossa after left vertebral artery injection. Scale bar $=10 \mathrm{~mm}$ 

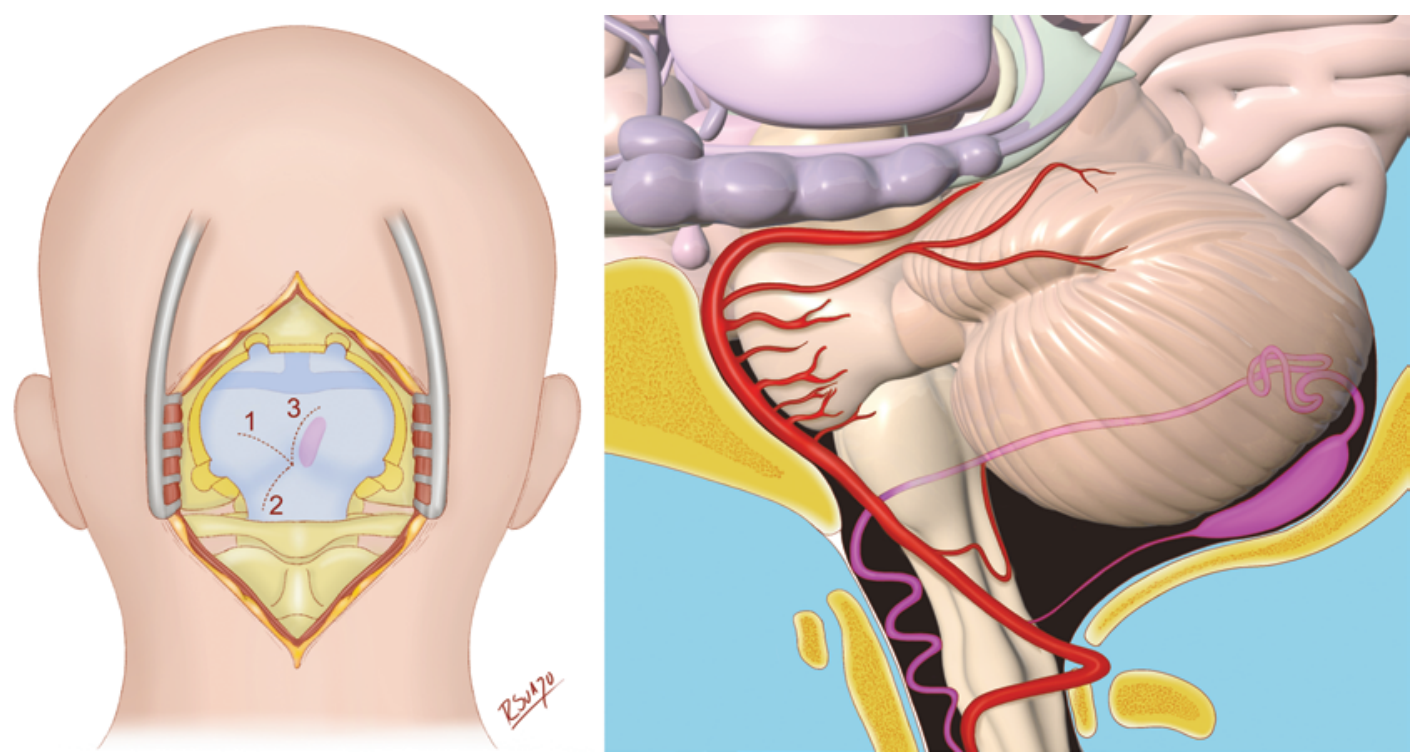

FIG. 3. Illustrations showing the incisions used for opening the dura (left) in a manner that allows visualization of the fistula and abnormal structures (right) so that they are not inadvertently sectioned. Artist: Roberto Sauzo. Copyright Toba N. Niazi. Published with permission.

outpatient rehabilitation. At the time, she still had a Foley catheter, although bowel and bladder sensation were improving, and she was unable to ambulate independently. One month after surgery, her bowel and bladder function had completely returned and she was able to walk with assistance. At the 1-year postoperative follow-up, the patient and family reported a complete return to baseline and MRI demonstrated resolution of spinal cord signal change (Fig. 6). Furthermore, follow-up catheter angiography 1 year after surgery showed no evidence of persistent fistula (not shown).

\section{Discussion}

To our knowledge, this is the first report of a Cognard Type V dAVF in a pediatric patient, although case reports and limited case series have described approximately 60 adult patients with these lesions. ${ }^{4-8}$ The 2 youngest patients reported prior to the current patient were a 20 -year-old woman and a 31-year-old man. ${ }^{5,7}$ Those patients also presented with acute/subacute tetraparesis, had no predisposing factors, were treated surgically, and made good recoveries. Our patient was unique in comparison to all previously reported adult patients with Cognard Type V dAVFs in that she had slowly progressive myelopathy that then acutely progressed over the course of a day to complete paralysis. Although we do not know what caused this acute deterioration, we assume that it was caused by a change in the hemodynamics of the fistula, leading to greater flow into the cortical veins and resultant edema. Nevertheless, we believe that recognition of Cognard Type V dAVF as a
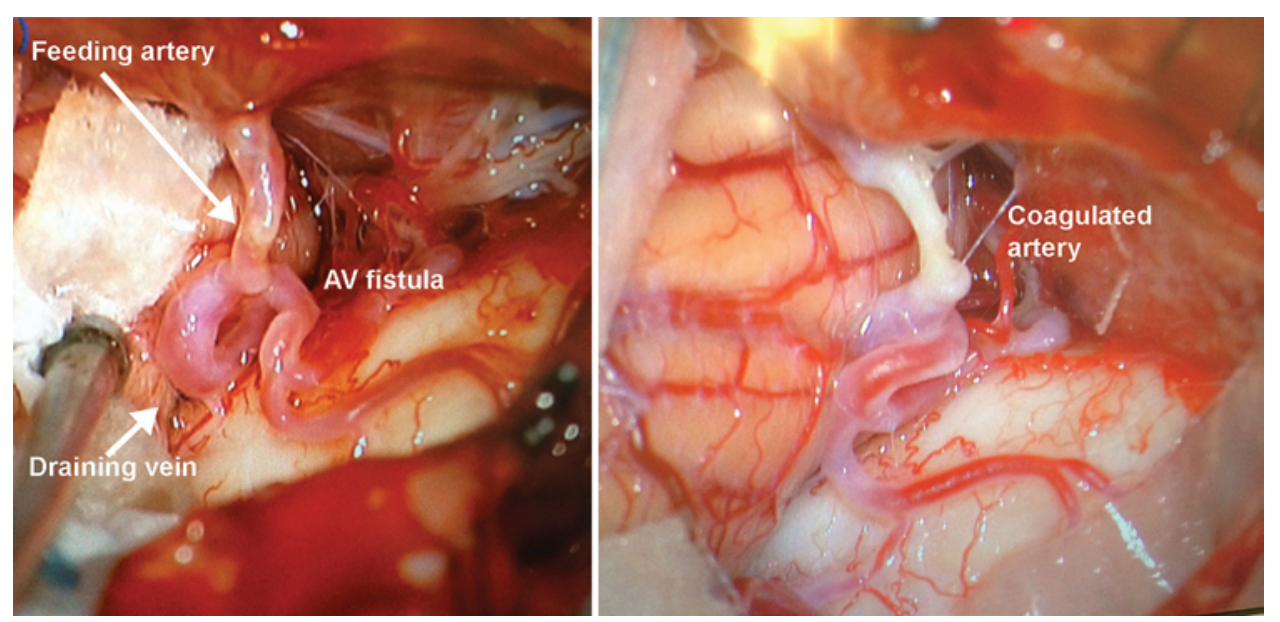

FIG. 4. Left: Intraoperative photograph of the dAVF with the feeding artery and the arterialized draining vein. Right: Intraoperative photograph showing the coagulated feeding artery and collapse of the arterialized draining vein. $A V=$ arteriovenous. Figure is available in color online only. 

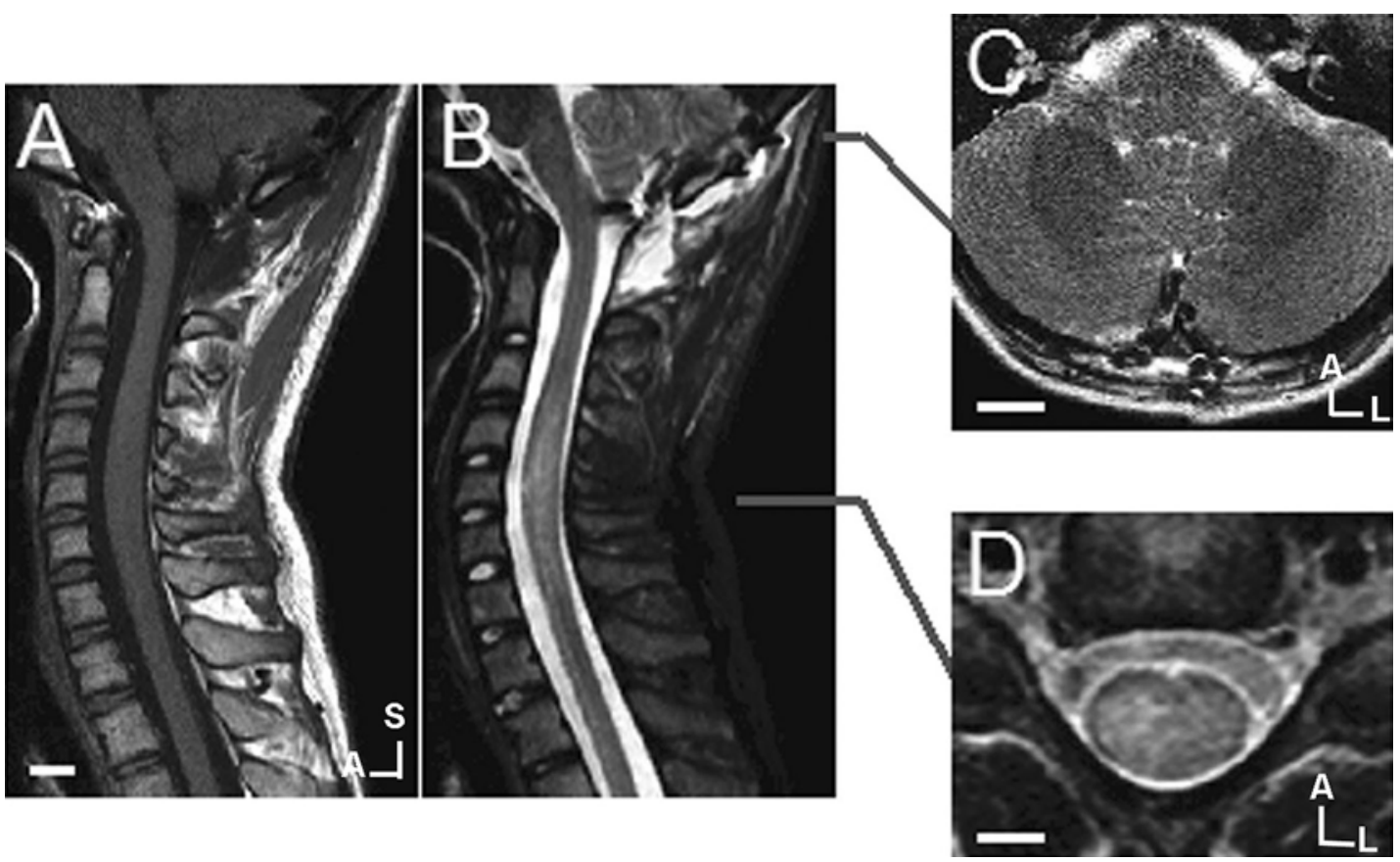

FIG. 5. Plain MR images obtained 6 days postoperatively. A: Sagittal T1-weighted image. Artifact from the surgical clips is visible in the posterior fossa. B: Sagittal T2-weighted image showing partial resolution of cervical cord edema and the complete absence of tortuous perimedullary veins visible prior to surgery. C: Axial T2 cut through the inferior cerebellum showing artifact from the surgical clips. No edema or significant blood products were noted. D: Axial T2 cut through the cervical cord. The gray lines show the dorsoventral levels of these axial cuts relative to those in panel B. Scale bar $=10 \mathrm{~mm}(A$ and $B) ; 4 \mathrm{~mm}(C$ and $D)$.

cause of rapidly progressive myelopathy is important, as it is a readily treatable condition that can result in significant morbidity if unrecognized.

\section{Diagnosis of Cognard Type V dAVFs}

Diagnosing Type V dAVFs solely based on clinical criteria is notoriously difficult because of the diverse clinical presentations that may mimic certain spinal cord pathologies. The progression of symptoms can occur over periods ranging from several hours to several years, with a median of 6 months, although 25\%-50\% of patients present with acute symptoms. ${ }^{7}$ Typical symptoms include ascending tetraparesis (62\%), sphincter dysfunction (34\%), and bulbar symptoms (31\%). The timing of symptom onset and constellation of symptoms do not appear to be related to long-term prognosis. ${ }^{5}$ The presentation and symptoms are often mistaken for other diagnoses, such as spinal stenosis, infarct, tumor, Guillain-Barré syndrome, or multiple sclerosis, although improved access to MRI and angiography have allowed for better detection and management. Roelz et al. ${ }^{9}$ recently reviewed MRI findings in 58 patients who presented with a Type V dAVF. Seventy-three percent of patients had central medullary edema on MRI, and $76 \%$ had T2 flow voids typical of engorged perimedullary veins. When contrast-enhanced MR angiography was used together with MRI, the detection rate of the perimedullary veins increased to $85 \%$. El Asri et al. ${ }^{5}$ suggested that the presence of brainstem signal abnormality on MRI, which was evident in 57\% of cases they reviewed, is an important prognostic factor and is correlated with worse prognosis. The brainstem T2 signal abnormality together with en- gorged perimedullary veins on MRI point toward a cranial dAVF rather than a spinal dAVF, which has similar clinical and MRI features but requires different management.

\section{Treatment of Cognard Type V dAVF}

Endovascular therapy has improved the multimodal treatment options available for Type V dAVF. The current recommendation for surgical treatment of Type V dAVFs, derived solely from adult patients, is to initially attempt endovascular therapy and then follow with open surgery if embolization is not successful. With this strategy, open surgery may be avoided if embolization is successful, but the risks associated with aggressive embolization in cases
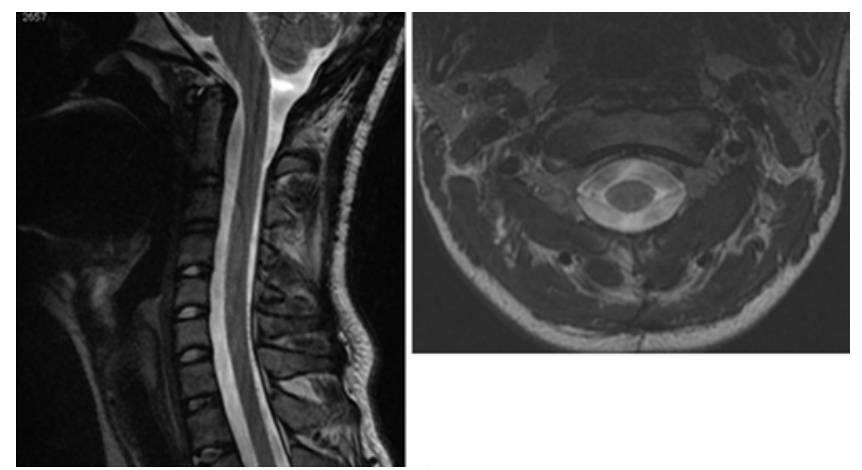

FIG. 6. Sagittal (left) and axial (right) T2-weighted MR images demonstrating resolution of the T2 signal cord change and engorgement of the cord with a lack of serpiginous spinal vessels at 1-year follow-up. 
with difficult vascular anatomy may also be avoided by having surgery as a backup option. In addition, as illustrated by our case, the anatomical information obtained from angiography may serve as an important tool for operative planning.

Several patient-specific factors need to be considered when planning each case. In infants, where blood loss is a greater risk than in adults and anatomy is more difficult to access, surgery may be a poorer option. Prior open surgical or failed embolic treatments would likely deter a surgeon from pursuing embolization. Furthermore, a smaller and more accessible fistula may be more amenable to treatment with open surgery than embolization, as was the case for our patient. Easy surgical access via an open posterior approach, combined with difficult endovascular access, made surgical clipping our treatment of choice for obliterating the fistula and reversing our patient's unique clinical course.

\section{Conclusions}

The diagnosis of Cognard Type V dAVF has historically been difficult. This is in part due to the discrepancy between the intracranial location of the fistula and symptoms localized to the spinal cord, but also because of the unpredictability of these lesions. This report complicates the picture by demonstrating that Type V dAVFs may present in children in a rapid, progressive manner. Thus, in children with unexplained myelopathy and/or MRI signal abnormalities in the cervical cord, suspicion for a Type V dAVF needs to be maintained. The presence of serpentine T1 or T2 flow voids on MRI is perhaps the most predictive radiographic clue to the presence of a Type V dAVF. When a dAVF is suspected, the workup for these fistulas requires both cranial and spinal angiography. We agree with other authors that endovascular embolization should be attempted if the vascular anatomy is amenable; however, for dAVFs with difficult endovascular access, favorable approach by open surgery, or prior failed embolization attempts, open surgical treatment with fistula ligation should be considered.

Generally, these cases are best treated by embolization. However, in cases in which the feeders are easy to catheterize but the cast of Onyx or glue used to treat the dAVF must occlude the entire fistula and the origin of the arterialized veins, embolization can often be difficult. A fistula is more amenable to surgery when there are only 1 or 2 arterialized veins connecting it to the pial or perimedullary veins. The aim of surgery then is to disconnect the fistula from the cortical or perimedullary veins.

\section{Acknowledgments}

We thank Robert Heros, MD, for reviewing the manuscript and Kristin Kraus, MSc, for editorial assistance.

\section{References}

1. Aixut Lorenzo S, Tomasello Weitz A, Blasco Andaluz J, Sanroman Manzanera L, Macho Fernández JM: Transvenous approach to intracranial dural arteriovenous fistula (Cognard V): a treatment option. A case report. Interv Neuroradiol 17:108-114, 2011 (Erratum in Interv Neuroradiol 18:114, 2012)

2. Cognard C, Gobin YP, Pierot L, Bailly AL, Houdart E, Casasco A, et al: Cerebral dural arteriovenous fistulas: clinical and angiographic correlation with a revised classification of venous drainage. Radiology 194:671-680, 1995

3. Crowley RW, Evans AJ, Jensen ME, Kassell NF, Dumont AS: Combined surgical/endovascular treatment of a complex dural arteriovenous fistula in 21-month-old. Technical note. J Neurosurg Pediatr 3:501-506, 2009

4. Duffau H, Lopes M, Janosevic V, Sichez JP, Faillot T, Capelle $\mathrm{L}$, et al: Early rebleeding from intracranial dural arteriovenous fistulas: report of 20 cases and review of the literature. J Neurosurg 90:78-84, 1999

5. El Asri AC, El Mostarchid B, Akhaddar A, Naama O, Gazzaz M, Boucetta M: Factors influencing the prognosis in intracranial dural arteriovenous fistulas with perimedullary drainage. World Neurosurg 79:182-191, 2013

6. Kincaid PK, Duckwiler GR, Gobin YP, Viñuela F: Dural arteriovenous fistula in children: endovascular treatment and outcomes in seven cases. AJNR Am J Neuroradiol 22:1217-1225, 2001

7. Li J, Ezura M, Takahashi A, Yoshimoto T: Intracranial dural arteriovenous fistula with venous reflux to the brainstem and spinal cord mimicking brainstem infarction-case report. Neurol Med Chir (Tokyo) 44:24-28, 2004

8. Natarajan SK, Ghodke B, Kim LJ, Hallam DK, Britz GW, Sekhar LN: Multimodality treatment of intracranial dural arteriovenous fistulas in the Onyx era: a single center experience. World Neurosurg 73:365-379, 2010

9. Roelz R, Van Velthoven V, Reinacher P, Coenen VA, Mader I, Urbach $\mathrm{H}$, et al: Unilateral contrast-enhancing pontomedullary lesion due to an intracranial dural arteriovenous fistula with perimedullary spinal venous drainage: the exception that proves the rule. J Neurosurg 123:1534-1539, 2015

\section{Disclosures}

The authors report no conflict of interest concerning the materials or methods used in this study or the findings specified in this paper.

\section{Author Contributions}

Conception and design: all authors. Acquisition of data: all authors. Drafting the article: all authors. Reviewed submitted version of manuscript: all authors. Approved the final version of the manuscript on behalf of all authors: Niazi.

\section{Correspondence}

Toba Niazi, Department of Pediatric Neurosurgery, University of Miami Miller School of Medicine, Nicklaus Children's Hospital, 3100 SW 62nd Ave., Miami, FL 33155. email: toba.niazi@mch. com. 